\title{
Outcome Measures of Chinese Herbal Medicine for Coronary Heart Disease: An Overview of Systematic Reviews
}

\author{
Jing Luo ${ }^{1,2}$ and Hao $\mathrm{Xu}^{2}$ \\ ${ }^{1}$ Graduate School, Beijing University of Chinese Medicine, Beijing 100029, China \\ ${ }^{2}$ Cardiovascular Diseases Center, Xiyuan Hospital, China Academy of Chinese Medical Sciences, Beijing 100091, China
}

Correspondence should be addressed to Hao Xu, xuhaotcm@gmail.com

Received 24 November 2011; Accepted 26 January 2012

Academic Editor: Keji Chen

Copyright (C 2012 J. Luo and H. Xu. This is an open access article distributed under the Creative Commons Attribution License, which permits unrestricted use, distribution, and reproduction in any medium, provided the original work is properly cited.

\begin{abstract}
Objective. The aim of this overview was to summarize the outcome measures of Chinese herbal medicine (CHM) as the treatment of coronary heart disease (CHD) based on available systematic reviews (SRs), so as to display the current situation and evaluate the potential benefits and advantages of CHM on CHD. Methods. An extensive search included the Cochrane Database of Systematic Reviews, MEDLINE, and 4 databases in Chinese. SRs of CHM for CHD were included. Besides evaluating and summarizing the outcome measures, we also estimated the quality of the included reviews by PRISMA (preferred reporting items for systematic reviews and meta-analyses). Data were extracted according to predefined inclusion criteria by two independent reviewers. Results. 46 articles were included. 20 kinds of CHM were reviewed. 7 SRs were concerned with myocardial infarction (MI), 38 SRs were related to angina pectoris. 11 SRs had primary endpoints, while others focused on secondary endpoints to evaluate CHM for CHD such as angina pectoris and electrocardiogram (ECG). One SR reported more adverse effects of CHM for CHD and of the SRs analyzed quality of life. Many CHM appeared to have significant effect on improving symptoms, ECG, biomarkers and so on. However, most SRs failed to make a definite conclusion for the effectiveness of CHM in CHD patients due specifically to the poor evidence. And according to PRISMA we found most of the trials in the SRs were of low quality. Conclusion. Primary endpoints were not used widely. The benefits of CHM for CHD need to be confirmed in the future with RCTs of more persuasive primary endpoints and high-quality SRs.
\end{abstract}

\section{Introduction}

Coronary heart disease (CHD) is the most common cause of death in western countries. With the infectious diseases controlled and improvement of people's living, the morbidity of CHD increases year by year in many developing countries. Acute myocardial infarction (AMI) and angina pectoris are the most important two types of CHD. Chinese herbal medicine (CHM) has a 3000-year-old history with unique theories for concepts of etiology and systems of diagnosis and treatment [1]. The interest in CHM is growing rapidly beyond China [2-5]. In recent years, some researchers have reported the effect of CHM on clinical symptoms, biomarkers and mortality in CHD patients. However, the evidence of CHM needs to be reviewed systematically and appraised critically.

High-quality systematic reviews (SRs) of randomized controlled trials (RCTs) are the sources of the best evidence
[6]. Currently, there is an increasing number of SRs on studies of CHM, but few of them concluded that $\mathrm{CHM}$ was definitely effective for CHD due to the weak evidence. In addition to rigorous clinical design and standard reporting, the selection of outcome measures also plays an important role in drawing a more persuasive conclusion. The aim of this overview was to summarize the outcome measures of $\mathrm{CHM}$ as the treatment of CHD based on available SRs, so as to display the current situation and evaluate the potential benefits and advantages of CHM on CHD.

\section{Methods}

Electronic literature searches were performed to identify the maximum possible number of systematic reviews/metaanalyses of $\mathrm{CHM}$ for $\mathrm{CHD}$. The following electronic databases were searched: (1) The Cochrane Database of 
Systematic Reviews (Issue 10 of 12, Oct 2011); (2) MEDLINE (2001 to 2011); (3) Chinese Biomedical Database (CBM, 2001 to 2011); (4) China National Knowledge Infrastructure (CNKI, 2001 to 2011); (5) Wanfang Databases (2001 to 2011); (6) Chinese VIP Information (VIP, 2001 to 2011). CBM, CNKI, Wanfang, and VIP were databases in Chinese. We searched databases in Chinese because CHMs were researched in china mostly. And we searched papers from 2001 to 2011 for high-quality RCTs and SRs mainly focusing in recent ten years.

The strategy below was used to search The Cochrane Library and adapted appropriately for use in different electronic bibliographic databases: \#1 herb*; \#2 medic*; \#3 (\#1 and \#2); \#4 Chinese; \#5 (\#3 or \#4); \#6 cardiac; \#7 heart; \#8 circulation; \#9 (\#6 or \#7 or \#8); \#10 (\#5 and $\# 9)$. To determine which article was we want, we scanned the title and abstract of each record independently by two reviewers ( $\mathrm{J}$. Luo and $\mathrm{H}$. Xu ). If the information included a systematic review or a meta-analysis of CHM for $\mathrm{CHD}$, the full paper was obtained for further assessment. Papers were excluded when problems occurred with: repeat publication; methodological studies; quality assessment report; the interventions in the control groups were other Chinese herbs; research on acupuncture, qigong, massage, or other treatments (Figure 1).

We divided the outcome measures into primary endpoints and secondary endpoints $[50,51]$. Primary endpoints include the mortality, AMI, restenosis after percutaneous coronary intervention (PCI), and recanalization. Secondary endpoints mainly indicate surrogate endpoints and laboratory measures, which include angina pectoris, arrhythmia, heart failure, consumption of nitroglycerine, electrocardiogram (ECG), ultrasonic cardiogram (UCG), Level of blood lipids, plasma endothelin, nitric oxide, myocardial enzyme, hemorheology, heart rate variability, and traditional Chinese medicine (TCM) syndrome.

In addition, we used PRISMA (preferred reporting items for systematic reviews and meta-analyses) as assessment tool to estimate the quality of the included reviews. This checklist includes 27 items of 7 key areas. And it describes the preferred way to present the abstract, introduction, methods, results, and discussion sections of a systematic review and a meta-analysis paper. It requires authors of each review to include a flow diagram that provides information about the number of studies identified, included, and excluded and the reasons for excluding them [52]. Information on each of the included reviews was imported into PRISMA statement for analysis. All data were extracted independently by two authors using predefined criteria. Disagreements were resolved by discussion between the authors. All inconsistencies were revised after a consensus was reached.

\section{Results}

46 articles were included (7 in English and 39 in Chinese). 39 SRs from the Chinese databases were published between 2004 and 2011. Since 2007, the number of SR increased markedly. 5 SRs from the Cochrane Database were published

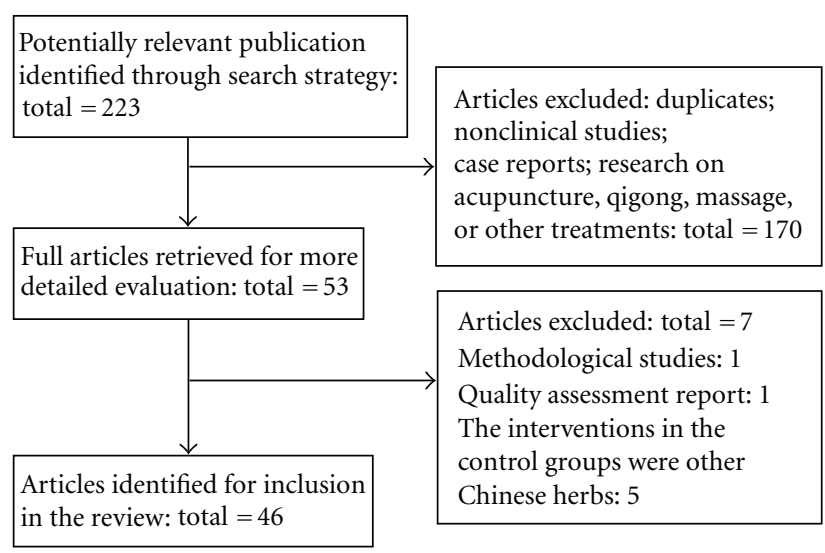

FIGURE 1: Flow-chart of SRs selection.

between 2006 to $2011[8,13,26,36,44]$. 2 SRs from MEDLINE were published between 2006 to 2011 [14, 45].

7 SRs were concerned with myocardial infarction (MI), 38 SRs were related to angina pectoris, and one SR was concerned with preventing and treating restenosis after PCI. The trials in SRs were mainly originated from china. The original trials included were called "RCTs" or "quasi-RCTs", but only a few of them were typical RCTs. Most of the trials in the SRs were of low quality, only 14 RCTs were high quality: one was concerned with MI, 12 were related to angina pectoris, and one was about preventing and treating restenosis.

20 kinds of CHM were reviewed, including injections, capsules, tablets, pellets, and herbal decoction as follows: Danshen preparations $(n=13)[8,14,20-22,33,38-41,45-$ 47], 7 of them were compound salvia pellet $[14,22,33,38$, 45-47]; Tongxinluo Capsule $(n=4)$ [13, 22, 27, 37]; Yiqi huoxue (supplementing qi and activating blood circulation) products $(n=3)[10,32,49]$; Xuefu zhuyu decoction $(n=2)$ $[23,30]$; herbal products $(n=4)[11,12,17,26]$; Shengmai injection $(n=2)[9,24]$; Suxiao jiuxin wan $(n=2)[35,36]$; Gingko $(n=2)[28,29]$; Acanthopanax $(n=2)[53,54]$; Puerarin $(n=2)[15,44]$; Shexiang baoxin wan $(n=2)$ $[34,55]$; Shenmai injection $(n=1)$ [7]; Tetramethylpyrazine $(n=1)$ [43]; Shuxuetong $(n=1)$ [48]; Xinkeshu $(n=1)$ [31]; Safflower injection $(n=1)$ [25]; Rhodiola $(n=1)$ [42]; Kudiezi injection $(n=1)$ [19]; Shuyu zaogan tablets $(n=1)$ [18]; Dengzhanhua injection $(n=1)$ [16].

11 SRs analyzed primary endpoints and the others all focused on secondary endpoints to evaluate CHM for CHD (Table 1). This was mainly based on whether there were available data in the original trials or not. Four primary endpoints were analyzed in the SRs including mortality, nonfatal myocardial infarction, restenosis after PCI, and recanalization. None of these SRs analyzed the quality of life. Angina pectoris was the most common secondary endpoint in the SRs. There was one SR without clear outcome measures [53], and 2 SRs only used "marked effective," "effective," "ineffective" as comprehensive outcome measures involving symptoms improvement and ECG changes [19, 54]. Many CHMs appear to have significant effect on improving symptoms, ECG, and level of blood lipids and 


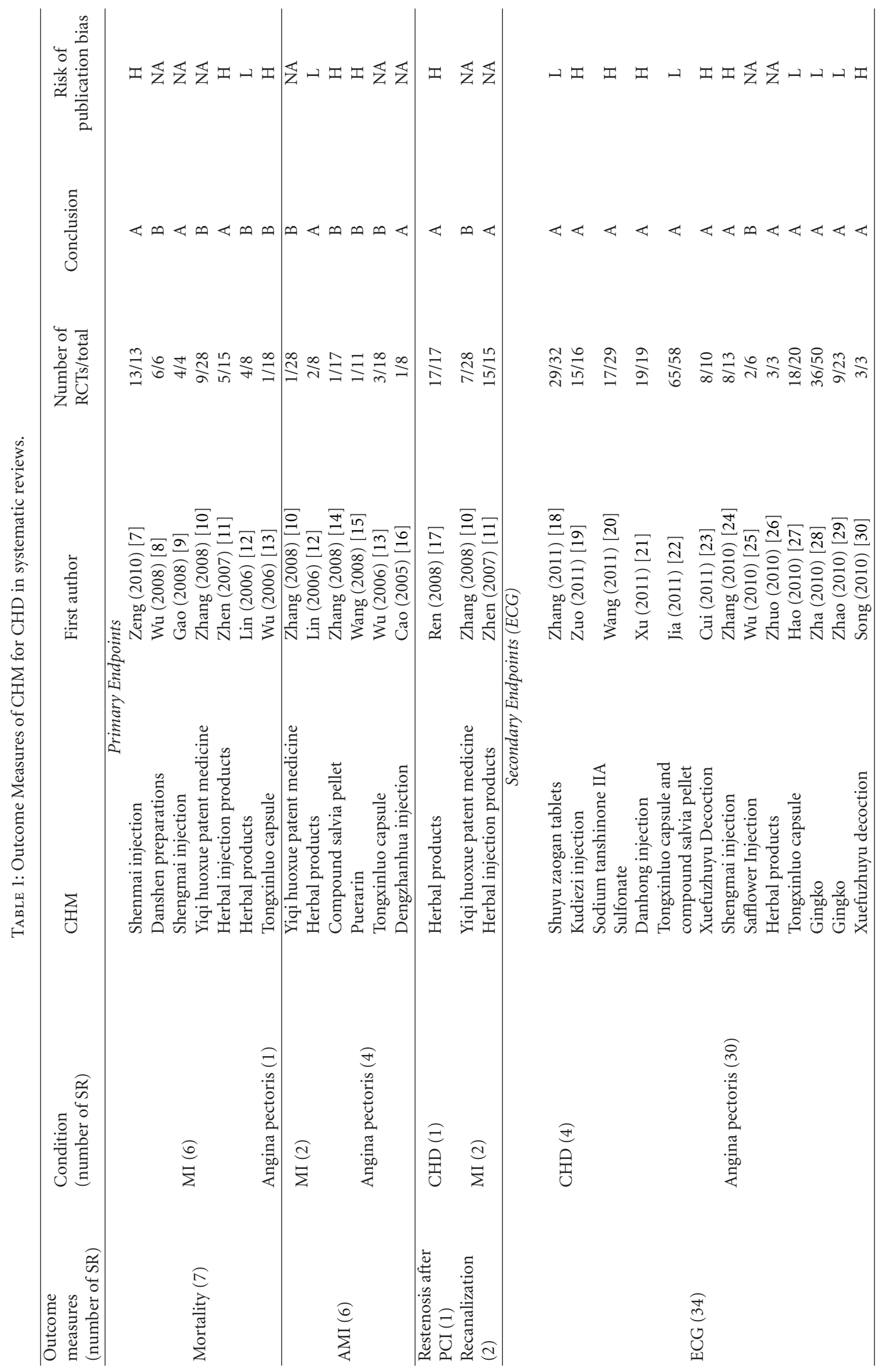




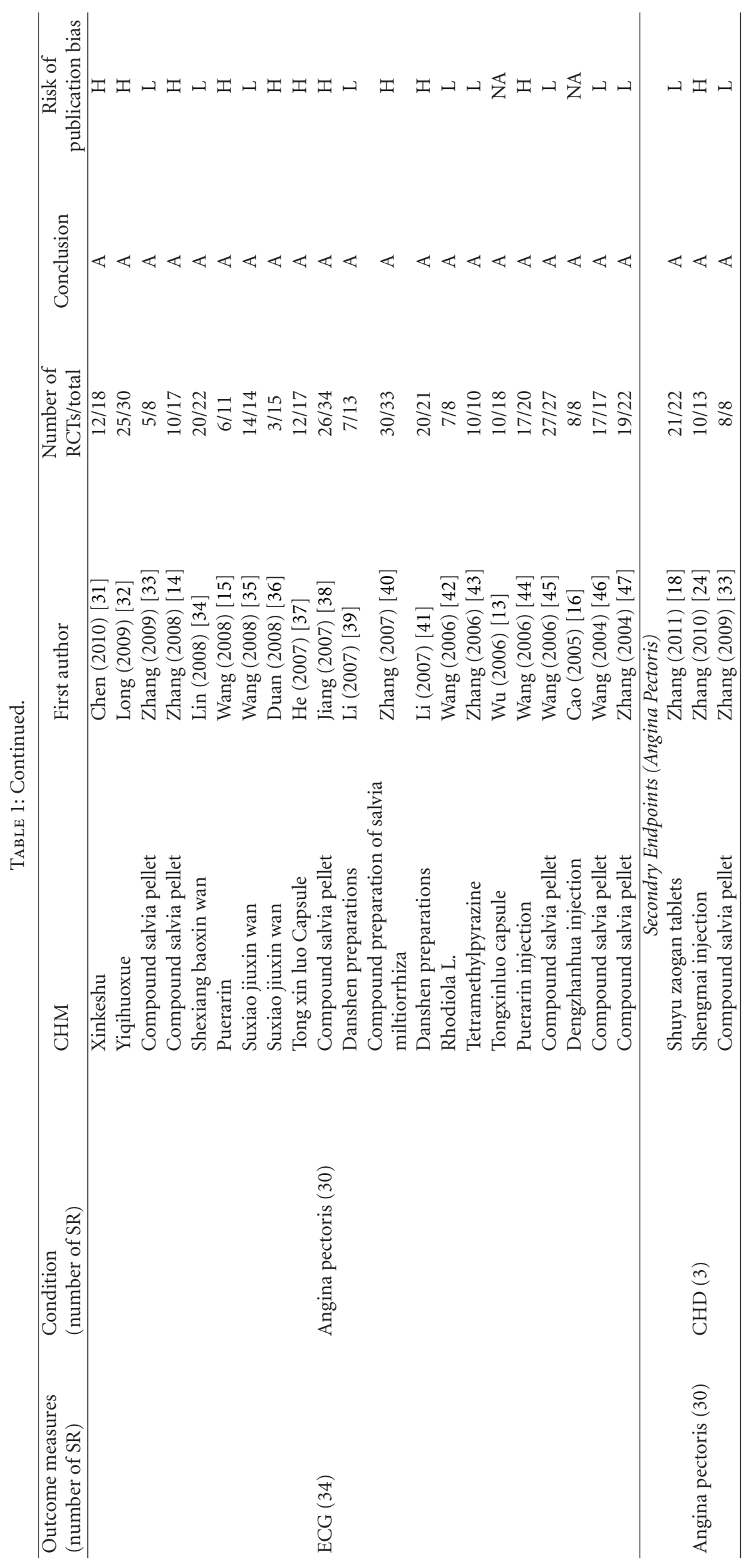




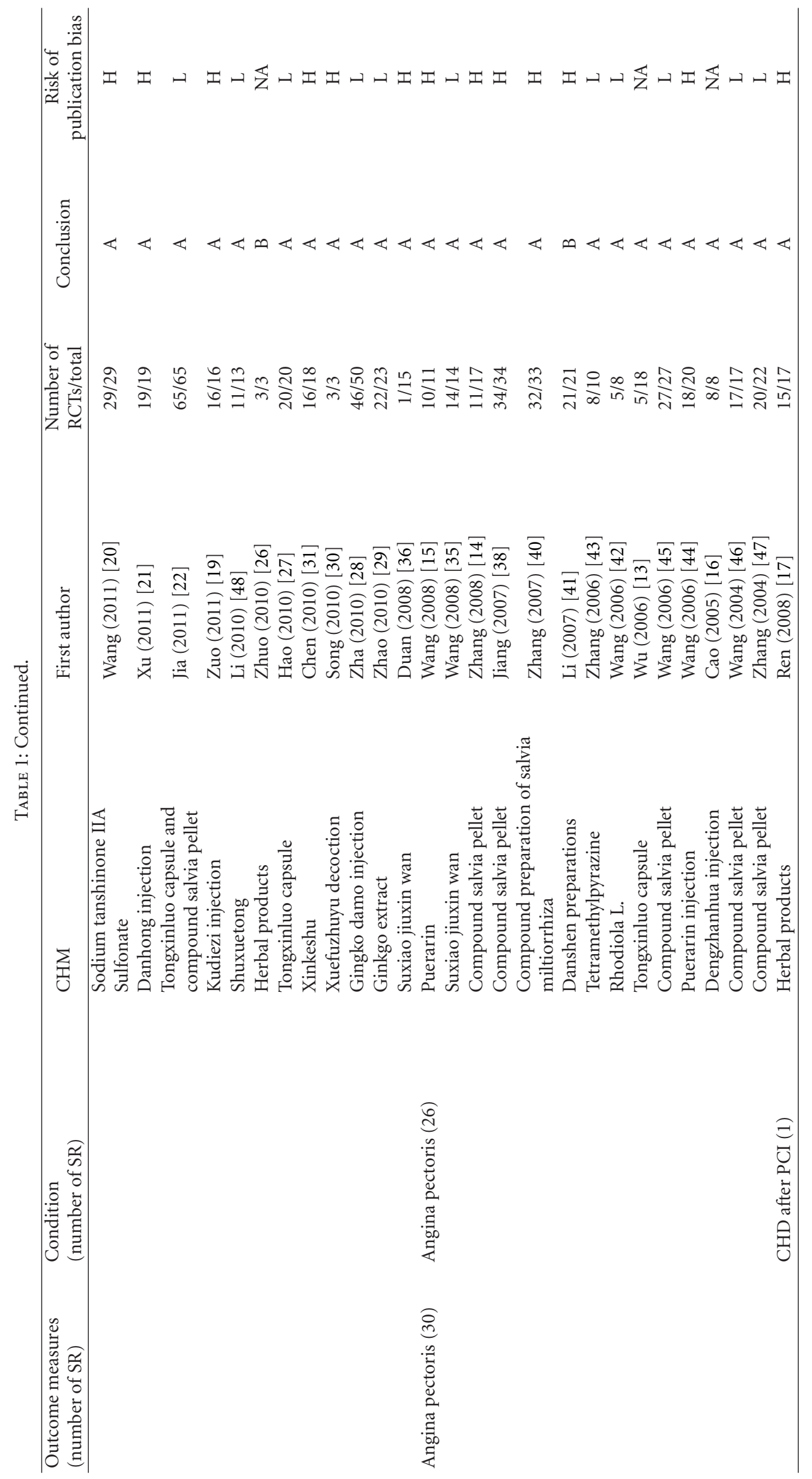




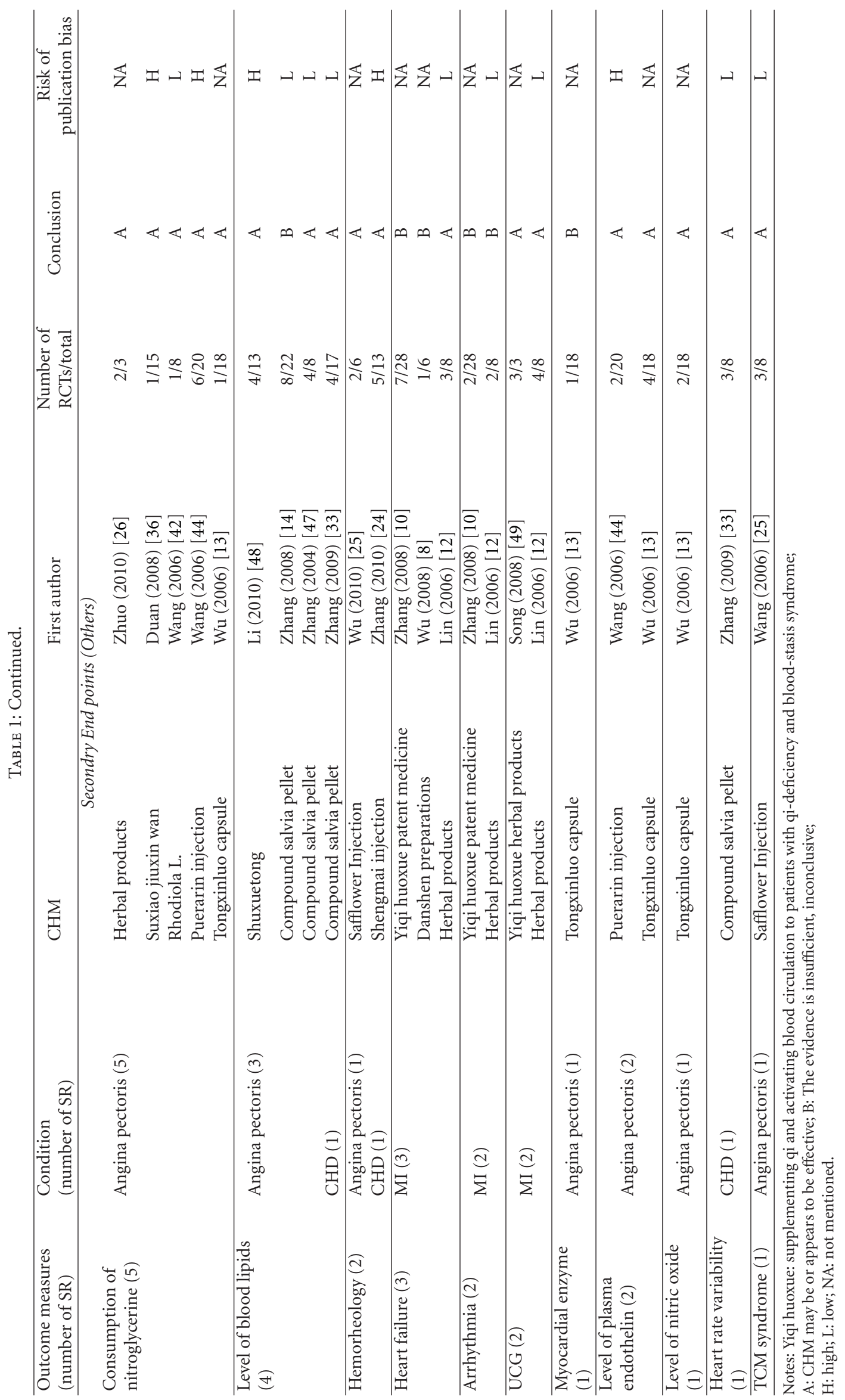


reducing the consumption of nitroglycerine, and so forth. Some SRs also reflected that CHM may be effective to reduce the risk of subsequent MI, heart failure, and arrhythmia. However, most SRs failed to draw a definite conclusion of the effectiveness of CHM for CHD due specifically to the poor evidence.

Adverse effects, which are important when evaluate a medicine, should be regarded as an essential outcome measure in clinical trials. However, only a few of the trials in the SRs had long-term data on adverse effects. Most of adverse effects of CHM were mentioned as "low adverse effect" or "none obvious". The adverse events reported majorly were abdominal complaints, nausea, and dyspepsia. One review reported more adverse reactions in treatment groups than in control groups [44]. Recently, several reviews have highlighted adverse reactions of CHM $[56,57]$.

Compared the usage of outcome measures between Cochrane and non-Cochrane reviews, we found that outcome measures of the included papers in Cochrane are more comprehensive. Every Cochrane review took primary endpoints, secondary endpoints, and safety as outcome measures. However, primary endpoints and safety are seldom taken as outcome measures in most of the non-Cochrane reviews. None of reviews analyzed quality of life or pay attention to medical economics.

According to PRISMA statement, we found that most of the included reviews are of low quality. The deficiencies are as follows: review methods in the abstracts and rationales for review were not well reported; only about half of the SRs reported the characteristics of included trials; just 5 SRs provided flow chart in the article, 2 in Chinese $[22,29]$, and 3 in English $[8,14,26]$; potential biases were not described well in the reports; most SRs lack in persuasive outcome measures.

\section{Discussion}

Our overview shows that primary endpoints and secondary endpoints are all used to evaluate the effect of CHM for CHD. Secondary endpoints are most commonly adopted in clinical trials due to their feasibility in small sample size and shortterm clinical trials. They may signify future cardiovascular event to some extent and are sure to be valuable as surrogate endpoints. But it is clearly that primary endpoints are more persuasive in RCT of cardiovascular diseases. However, most of the outcome measures in the included SRs are angina pectoris and ECG. Primary endpoints such as mortality and major cardiovascular events are not used widely. Adverse effects, quality of life, and medical economics, which are also important when evaluate a medicine, should be taken as outcome measures too. All of these are the reasons why neither the trials nor the SRs of CHM for CHD could meet a sufficiently high standard to be broadly accepted by the Western medical community.

SRs of CHM with poor methodology and reporting quality have been reported [58]. According to PRISMA statement, we found that most of the included reviews have poor quality. Reviewers were not good at reporting how they avoided bias in selecting primary studies, how they extracted data, and how they evaluated the validity of the primary studies. Also, most of the reviewers chose less persuasive outcome measures, which reduced the persuasion of the interventions. So if reviewers did not master the method of performing SR, they could produce inaccurate or misleading conclusions for current clinical practice and even the future research. Although it appeared that CHM was effective for CHD in clinical use, such as compound salvia pellet, shengmai injection, suxiao jiuxin wan, and gingko, puerarin, most SRs were inconclusive that CHM had a definite effect for $\mathrm{CHD}$ owing to the poor evidence.

Before recommending the conclusion, we have to consider the following weaknesses in this overview. Firstly, data were abstracted from SRs instead of the original trials, and most of the included SRs have poor quality. Secondly, most of the RCTs in the SRs included are also of low quality due mainly to unclear randomization and blinding method, incomplete outcome reporting, publication bias, and so forth. Thirdly, we only selected SRs published in Chinese and English. SRs of CHM for CHD published in other language or originated from other countries might be omitted. Fourthly, we did not identify unpublished studies, thus negative trial might not be reported and could induce publication bias.

In conclusion, primary and secondary endpoints were all used to evaluate the effectiveness of CHM for CHD, but primary endpoints were not used widely. Although it appeared that CHM was effective for CHD in terms of some outcome measures, most SRs failed to draw a definite conclusion for the effectiveness of CHM in CHD patients due to the poor evidence. The benefits of CHM for CHD still need to be confirmed in the future with RCTs of more persuasive primary endpoints and high-quality SRs.

\section{References}

[1] K. J. Chen and H. Xu, "The integration of traditional Chinese medicine and Western medicine," European Review, vol. 11, no. 2, pp. 225-235, 2003.

[2] N. Robinson, "Integrative medicine-traditional Chinese medicine, a model?" Chinese Journal of Integrative Medicine, vol. 17, no. 1, pp. 21-25, 2011.

[3] G. Dobos and I. Tao, "The model of western Integrative medicine: the role of Chinese medicine," Chinese Journal of Integrative Medicine, vol. 17, no. 1, pp. 11-20, 2011.

[4] H. A. Tindle, R. B. Davis, R. S. Phillips, and D. M. Eisenberg, "Trends in use of complementary and alternative medicine by US adults: 1997-2002," Alternative Therapies, Health and Medicine, vol. 11, no. 1, pp. 42-49, 2005.

[5] D. Eisenberg, "Reflections on the past and future of integrative medicine from a lifelong student of the integration of Chinese and western medicine," Chinese Journal of Integrative Medicine, vol. 17, no. 1, pp. 3-5, 2011.

[6] Medical Research Library of Brooklyn, "The Evidence Pyramid," http://library.downstate.edu/EBM2/2100.htm.

[7] Y. J. Zeng, J. Wang, Y. C. Zhou, and M. X. Huang, "Effect of shenmai injection on mortality rate of patients with acute myocardial infartion: a systematic review," Modern Journal of 
Integrated Traditional Chinese and Western Medicine, vol. 19, no. 28, pp. 3555-3558, 2010.

[8] T. Wu, J. Ni, and J. Wu, "Danshen (Chinese medicinal herb) preparations for acute myocardial infarction," Cochrane Database of Systematic Reviews, no. 2, article CD004465, 2008.

[9] Z. Y. Gao, C. Y. Guo, D. Z. Shi, K. J. Chen, H. Xu, and T. X. Guo, "Effect of Shengmai injection on the fatality rate of patients with acute myocardial infarction: a Systematic review," Chinese Journal of Integrated Traditional and Western Medicine, vol. 28, no. 12, pp. 1069-1073, 2008.

[10] J. H. Zhang, H. C. Shang, B. L. Zhang, X. M. Gao, Y. Z. Xiang, and H. Wang, "Systematic review of randomized controlled trials on treatment of myocardial infarction with YiqiHuoxue Chinese Patent medicine," China Journal of Traditional Chinese Medicine and Pharmacy, vol. 23, no. 4, pp. 300-306, 2008.

[11] L. Zhen, H. X. Liu, and J. J. Shang, "Effect of herbal injection products as adjutant treatment on mortality rate and recanalization rate of patients with acute myocardial infartion: a Meta Analysis," Journal of Emergency in Traditional Chinese Medicine, vol. 16, no. 7, pp. 859-862, 2007.

[12] Q. Lin, Y. B. Nong, and W. H. Duan, "Meta analysis of clinical research literature on Chinese materia medica for acute myocardial infarction," China Journal of Traditional Chinese Medicine and Pharmacy, vol. 21, no. 9, pp. 529-55631, 2006.

[13] T. Wu, R. A. Harrison, X. Chen et al., "Tongxinluo (Tong xin luo or Tong-xin-luo) capsule for unstable angina pectoris," Cochrane database of systematic reviews (Online), no. 4, article CD004474, 2006.

[14] J. H. Zhang, H. C. Shang, X. M. Gao et al., "Compound salvia droplet pill, a traditional Chinese medicine, for the treatment of unstable angina pectoris: a systematic review," Medical Science Monitor, vol. 14, no. 1, pp. RA1-RA7, 2008.

[15] J. Wang, Y. Tian, C. Feng, and H. Sheng, "Effect of Puerarin injection for unstable angina pectoris: a Systematic Review," Guangming Journal of Chinese Medicine, vol. 23, no. 4, pp. 399403, 2008.

[16] W. Cao, D. Lan, T. Zhang, C. Tang, T. X. Wu, and G. J. Liu, "Effect of Dengzhanhua Injection for angina pectoris: a systematic review," Chinese Journal of Evidence-Based Medicine, vol. 5, no. 4, pp. 317-322, 2005.

[17] Y. Ren, K. J. Chen, and X. M. Ruan, "Systematic review of randomized controlled trials on preventing and treating restenosis after percutaneous coronary intervention with Chinese medicine," Chinese Journal of Integrated Traditional and Western Medicine, vol. 28, no. 7, pp. 597-601, 2008.

[18] Z. H. Zhang, Z. J. Chen, Y. C. Sheng et al., "Systematic evaluation of Shuyu Zaogan tablets for coronary heart disease," World Clinical Drugs, vol. 32, no. 3, pp. 159-164, 2011.

[19] Z. J. Zuo and Q. M. Huang, "A systematic review of Kudiezi injection in the treatment of angina pectoris," China Medical Herald, vol. 8, no. 4, pp. 32-35, 2011.

[20] C. 1. Wang, F. Gu, and S. L. Wang, "Meta-analysis on randomized controlled trials for treatment of angina pectoris by sodium tanshinone IIA sulfonate," Chinese Journal of Integrative Medicine on Cardio/Cerebrovascular Disease, vol. 9, no. 6, pp. 644-647, 2011.

[21] G. L. Xu, S. M. Lin, H. Xu, and L. Qin, "Meta-analysis of Danhong injection for unstable angina pectoris," Li Shizhen Medicine and Materia Medica Research, vol. 22, no. 3, pp. 765767, 2011.

[22] Y. L. Jia, S. K. Zhang, F. F. Bao, F. Y. Huang, and S. W. Liang, "Indirect Comparison of Tongxinluo capsule and Danshen dripping pill for angina pectoris: a Systematic Review," Chinese Journal of Evidence-Based Medicine, vol. 11, no. 8, pp. 919-931, 2011.

[23] H. J. Cui, H. Y. He, and Z. H. Xing, "System Evaluation and Meta Analysis of Xuefuzhuyu Decoction on unstable angina pectoris," Journal of Emergency in Traditional Chinese Medicine, vol. 20, no. 7, pp. 1071-1074, 2011.

[24] Q. Zhang and R. M. Jin, "Meta-analysis of Shengmai injection and conventional therapy for coronary heart disease," Chinese Journal of New Drugs and Clinical Remedies, vol. 29, no. 4, pp. 310-314, 2010.

[25] F. B. Wu, T. Xu, J. Li, and Y. Tang, "Meta Analysis on Efficacy and Safety of Safflower Injection in Treating unstable angina pectoris," China Pharmaceuticals, vol. 19, no. 17, pp. 4-5, 2010.

[26] Q. Zhuo, Z. Yuan, H. Chen, and T. Wu, "Traditional Chinese herbal products for stable angina," Cochrane database of systematic reviews (Online), vol. 5, article CD004468, 2010.

[27] C. H. Hao and J. Y. Zhang, "Meta-analysis of Tongxinluo Capsule for coronary heart disease angina," China Modern Docter, vol. 48, no. 14, pp. 6-9, 2010.

[28] Y. Zha and L. Li, "Systematic Evaluation of Yinxing Damo injection in the treatment of angina pectoris," China Pharmacy, vol. 21, no. 44, pp. 4143-4147, 2010.

[29] W. Zhao, J. S. Xiang, and K. Ye, "Systematic Review on Randomized Controlled Trials for treatment of UAP by Ginkgo extract," Journal of Liaoning University of Traditional Chinese Medicine, vol. 12, no. 11, pp. 216-220, 2010.

[30] X. M. Song, "Effect of Xuefuzhuyu decoction as adjutant treatment to isosorbide dinitrate and aspirin for coronary angina pectoris: a Meta-analysis," The Journal of Practical Medicine, vol. 26, no. 14, pp. 2633-2635, 2010.

[31] X. T. Chen, S. E. Guo, and Y. Guo, "Effect of Xinkeshu for coronary angina pectoris: a Meta-analysis," Journal of Changchun University of Traditional Chinese Medicine, vol. 26, no. 3, pp. 357-359, 2010.

[32] Y. Long, X. Jin, Z. J. Shao, J. Ma, J. H. Li, and T. H. Xiao, "Systematic review on randomized controlled trials for qisupplementing and blood-quickening treatment of angina pectoris due to qi-deficiency and blood stasis," China Heart Journal, vol. 21, no. 1, pp. 54-59, 2009.

[33] M. Z. Zhang and J. Zhang, "Meta-analysis of Compound salvia pellet for coronary heart disease," Li Shizhen Medicine and Materia Medica Research, vol. 20, no. 4, pp. 1007-1008, 2009.

[34] H. Lin and W. P. Tang, "Systematic Review of Randomized Controlled Trials on treating CHD with Shexiang baoxin wan," First National Middle-Aged MD Forum Proceedings of Integrative Medicine on Cardiovascular Disease, pp. 147-152, 2008.

[35] X. J. Wang and B. N. Xu, "Meta Analysis on treating angina pectoris with Suxiao jiuxin pill," Shaanxi Journal of Traditional Chinese Medicine, vol. 29, no. 9, pp. 1249-1251, 2008.

[36] X. Duan, L. Zhou, T. Wu et al., "Chinese herbal medicine suxiao jiuxin wan for angina pectoris," Cochrane Database of Systematic Reviews, no. 1, article CD004473, 2008.

[37] S. Z. He, W. K. Wu, Z. S. Deng, S. Y. Ou, and S. F. Liu, "Systematic Review on Randomized Controlled Trials for treatment of coronary heart disease by Tong xin luo Capsul," Journal of Sun Yat-Sen University, vol. 28, no. 5, pp. 573-577, 2007.

[38] S. Y. Jiang, J. C. Tong, R. Y. Shun, and H. T. Xie, "Meta-analysis of Compound salvia pellet for coronary angina pectoris," Practical Pharmacy and Clinical Remedies, vol. 10, no. 6, pp. 334-337, 2007. 
[39] K. J. Li, "A systematic review on Randomized Controlled Trials for treatment of unstable angina pectoris with Danshen preparations," Guangming Journal of Chinese Medicine, vol. 22, no. 2, pp. 37-40, 2008.

[40] J. H. Zhang, H. C. Shang, X. M. Gao et al., "Systematic evaluation of Compound preparation of salvia miltiorrhiza in treating stable angina in a randomized controlled trial," Tianjin Journal of Traditional Chinese Medicine, vol. 24, no. 3, pp. 195-200, 2007.

[41] K. J. Li, "A systematic review on randomized controlled trials for treatment of stable angina pectoris with Danshen preparations," Herald of Medicine, vol. 26, no. 4, pp. 383-386, 2007.

[42] X. Wang, Y. Y. Zhu, and L. S. Hu, "A Systematic review of Rhodiola L. for treating angina," Progress in Modern Biomedicine, vol. 6, no. 2, pp. 42-45, 2006.

[43] Y. C. Zhang, F. C. Zhi, Q. X. Tan, and J. He, “Treating unstable angina with tetramethylpyrazine: a systematic review," Chinese Journal of Clinical Rehabilitation, vol. 10, no. 27, pp. 102-104, 2006.

[44] Q. Wang, T. Wu, X. Chen et al., "Puerarin injection for unstable angina pectoris," Cochrane Database of Systematic Reviews, vol. 3, article CD004196, 2006.

[45] G. Wang, L. Wang, Z. Y. Xiong, B. Mao, and T. Q. Li, "Compound salvia pellet, a traditional Chinese medicine, for the treatment of chronic stable angina pectoris compared with nitrates: a meta-analysis," Medical Science Monitor, vol. 12, no. 1, pp. SR1-SR7, 2006.

[46] L. Wang, Z. Y. Xiong, and G. Wang, "Systematic assessment on randomized controlled trials for treatment of stable angina pectoris by compound salvia pellet," Chinese Journal of Integrated Traditional and Western Medicine, vol. 24, no. 6, pp. 500-504, 2004.

[47] M. Z. Zhang, L. Wang, B. J. Chen, and G. N. Nie, "Metaanalysis of Document on Compound Danshen dropping pills (DSP) in Treatment of patients with Stable Angina," Chinese Journal of Integrative Medicine Cardio/Cerebrovascular Disease, vol. 2, no. 6, pp. 311-314, 2004.

[48] X. Y. Li, F. Du, W. L. Cheng, and H. Xia, "Systematic review on randomized controlled trials for treatment of unstable angina pectoris by Shuxuetong," Modern Journal of Integrated Traditional Chinese and Western Medicine, vol. 19, no. 18, pp. 2231-2233, 2010.

[49] Q. G. Song, W. X. Du, M. Liu et al., "Effect of Yiqi Huoxue herbal products for ventricular remodeling after acute myocardial infarction: a Meta-analysis," Liaoning Journal of Traditional Chinese Medicine, vol. 35, no. 3, pp. 323-325, 2008.

[50] European Medicines Agency (EMA), "Committee for medicinal products for human use, Guideline on the evaluation of medicinal products for cardiovascular Disease prevention London, 2008," http://www.ema.europa.eu/ema/index/.

[51] S. Yusuf, S. Mehta, S. Anand et al., "The Clopidogrel in Unstable angina to prevent Recurrent Events (CURE) trial programme: rationale, design and baseline characteristics including a meta-analysis of the effects of thienopyridines in vascular disease," European Heart Journal, vol. 21, no. 24, pp. 2033-2041, 2000.

[52] D. Moher, A. Liberati, J. Tetzlaff, and D. G. Altman, "The PRISMA Groupl. Preferred Reporting Items for Systematic Reviews and Meta-analyses:The PRISMA statement," PLOS Med, vol. 6, no. 7, article e1000097, 2009.

[53] P. Wang, Y. G. Xu, and Z. Y. Gao, "Effect of Acanthopanax Senticosus injection for coronary angina pectoris: a Meta- analysis," Lishizhen Medicine and Materia Medica Research, vol. 18, no. 9, pp. 2243-2244, 2007.

[54] Z. H. Zhong and W. H. Li, "A Meta Analysis on Randomized Controlled Trials for treatment of coronary angina pectoris with Acanthopanax Senticosus injection," China Pharmaceuticals, vol. 16, no. 15, pp. 5-6, 2007.

[55] X. G. Zhou, H. W. Wang, and G. B. Yu, "Meta Analysis on treating unstable angina pectoris with Shexiang baoxin wan," Chinese Traditional Patent Medicine, vol. 26, supplement, pp. $1-6,2004$.

[56] L. M. Guo and Y. G. Feng, "Adverse reaction of puerarin injection," Chinese Magazine of Clinical Medicinal Professional Research, no. 69, pp. 11495-11497, 2003.

[57] L. M. Zhi and Z. Q. Zhang, "Review on the adverse reactions of puerarin injection," Hebei Traditional Chinese Medicine, vol. 24, no. 7, pp. 555-556, 2002.

[58] J. H. Zhang, H. C. Shang, X. M. Gao et al., "Methodology and reporting quality of systematic review/meta-analysis of traditional Chinese medicine," Journal of Alternative and Complementary Medicine, vol. 13, no. 8, pp. 797-805, 2007. 


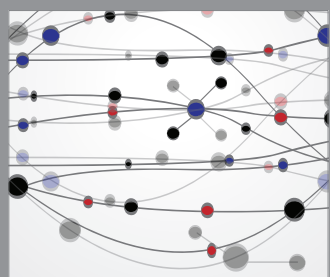

The Scientific World Journal
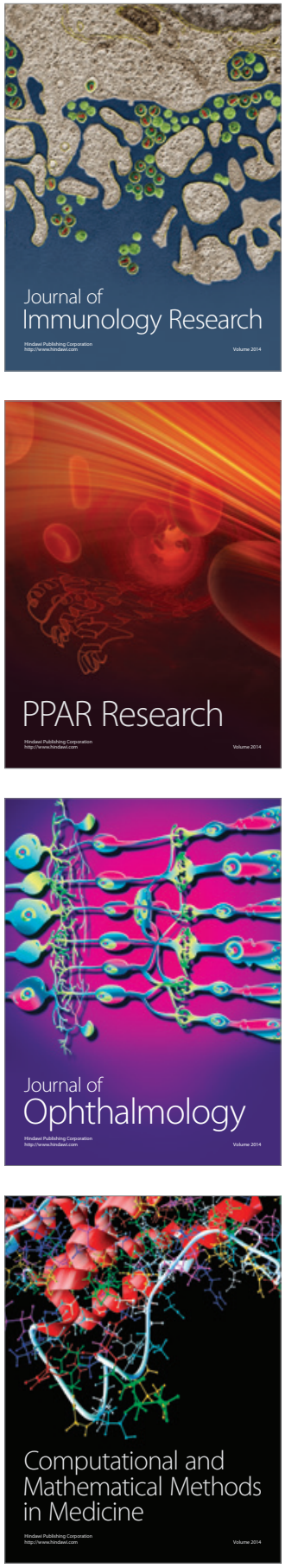

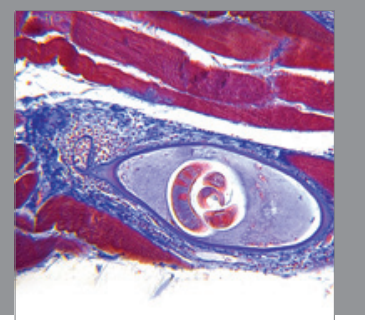

Gastroenterology

Research and Practice
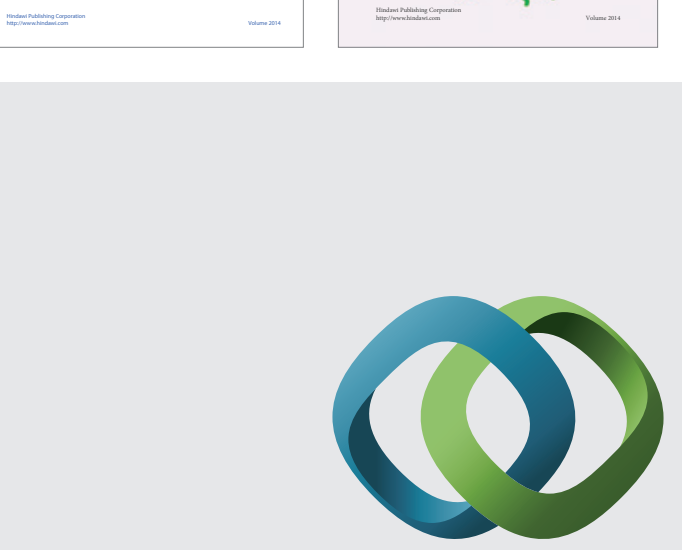

\section{Hindawi}

Submit your manuscripts at

http://www.hindawi.com
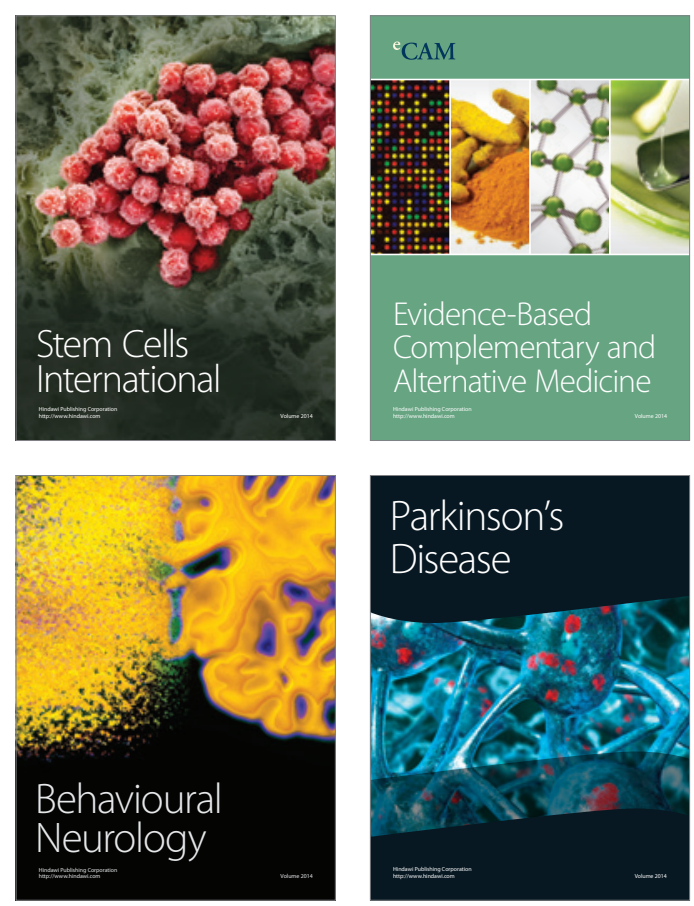

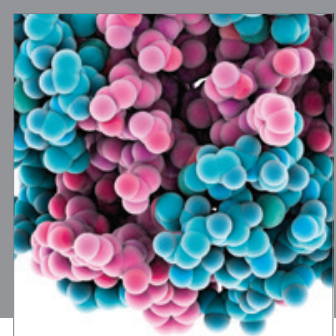

Journal of
Diabetes Research

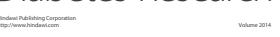

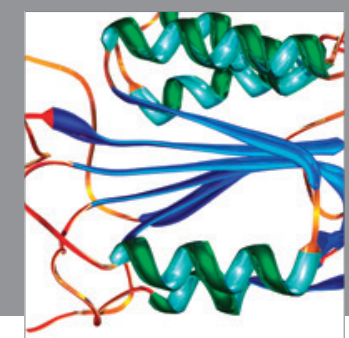

Disease Markers
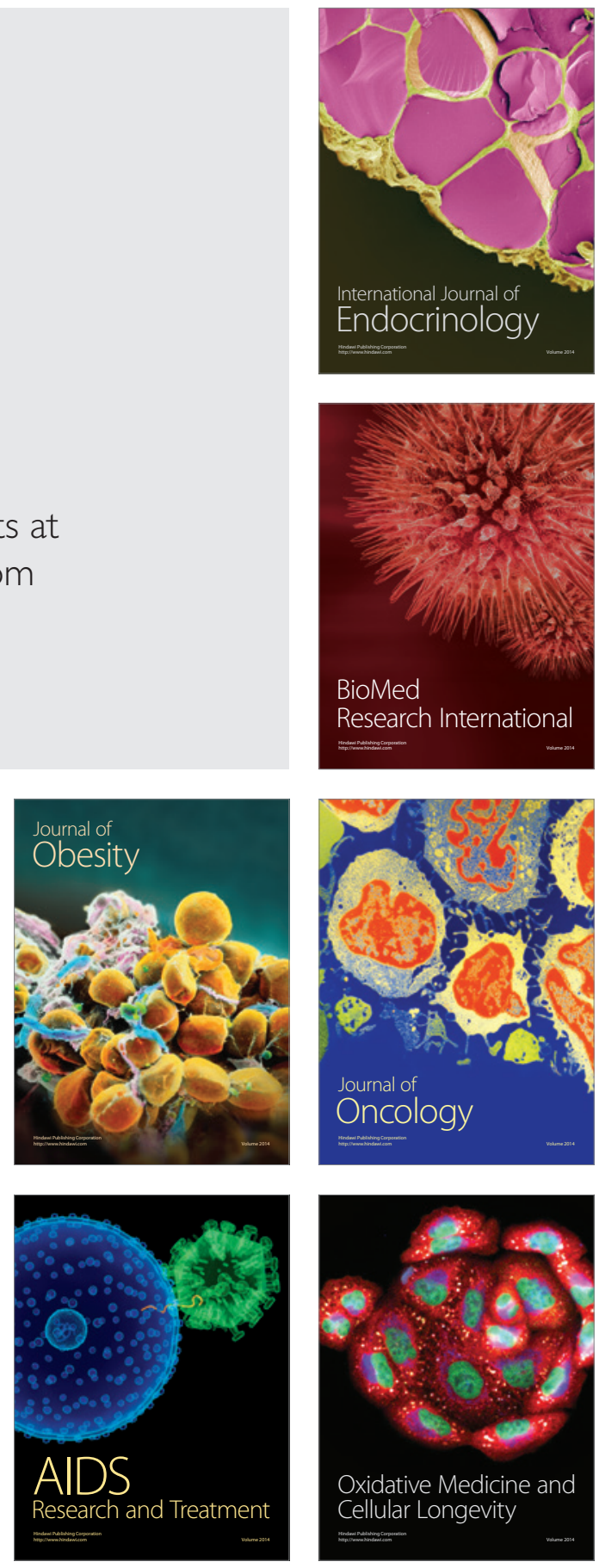